\title{
Serological testing for celiac disease in adults
}

United European Gastroenterology Journal

\author{
Astrid Collatz Schyum and Jüri Johannes Rumessen
}

DOI: $10.1177 / 2050640613502788$

ueg.sagepub.com

@SAGE

\begin{abstract}
Background and aim: We present a systematic review on the performance of currently available methods for serological diagnosis of celiac disease (CD) and the role of human leukocyte antigen (HLA) typing.

Objective: A literature survey was conducted using PubMed, MeSH database, Web of Science as well as manual searches. Results: Tissue transglutaminase antibodies (tTG) (IgA) (tested in nine studies) show sensitivities and specificities in the range of $0.76-0.968$ and $0.909-0.98$, and deamidated gliadin peptide (DGP) (IgA and IgG) (tested in eight studies) show sensitivities and specificities in the range of 0.69-0.984 and 0.903-1. Endomysial antibodies (EMA) (tested in five studies) show sensitivities and specificities in the range of $0.61-0.937$ and $0.98-1$, respectively. Combination assays (tested in three studies) using DGP + tTG and DGP (IgA + IgG) show sensitivities and specificities in the range of $0.87-1$ and $0.8-1$, respectively. HLA DQ2/DQ8 may be necessary for the development of CD-HLA DQ2 in particular. A possible close correlation may also exist between CD and HLA-G.

Conclusion: DGP and tTG for serological testing for CD show equivalent diagnostic performance. More studies with, in particular, DGP alone and in combination with ITG are necessary before a firm recommendation can be made. HLA typing to exclude CD may still be controversial. It still seems premature to diagnose celiac disease in adults based on serology alone.
\end{abstract}

Keywords

Celiac disease, diagnosis, serological testing, HLA DQ2/DQ8, gliadin, transglutaminase

Received: 14 June 2013; accepted: 2 August 2013

\section{Introduction}

Celiac disease $(\mathrm{CD})$ is an autoimmune, gluten-sensitive enteropathy where intake of foods containing gluten, a protein found in grain species such as wheat, barley and rye, to cause mucosal damage in the small intestine, leading to malabsorption. ${ }^{1}$

Ninety percent of all patients with celiac disease carry HLA DQ2 and probably all of the remaining $10 \%$ carry HLA DQ8. However, these haplotypes are carried by about $30 \%$ of the unaffected population as well. ${ }^{2}$ The prevalence of $\mathrm{CD}$ is estimated to be around $1 \%$ in the general population. ${ }^{3}$ It is assumed that HLA DQ2/DQ8 represents less than $50 \%$ of the genetic explanation of CD's etiology, ${ }^{4,5}$ which means that there must be other genetic factors playing a role in disease development. It is still controversial whether HLA DQ2 or DQ8 is necessary for the development of CD.

The area of serological testing for CD has developed greatly in recent years. The most commonly used antibodies are antigliadin antibodies (AGA), endomysial antibodies (EMA) and tissue transglutaminase antibodies (tTG). ${ }^{6,7}$ The AGA test was developed in the early 1980s, and more recently developed assays may have higher sensitivities and specificities than AGA, which is considered obsolete. ${ }^{8-13}$ The recently introduced deamidated gliadin peptide antibody (DGP) has shown promising performance as compared to EMA and tTG test results. ${ }^{6-9,13-15}$ Data and experience concerning the usage of DGP are still preliminary, however.

EMA was developed in the mid-1980s after AGA and has generally shown high specificity and sensitivity. However, drawbacks of this assay are the resourcedemanding use of immunofluorescence and the operator-dependent nature of the test results, possibly diminishing the objectivity of the test. ${ }^{8,9,16}$ The tTG assay, which uses enzyme-linked immunosorbent assay (ELISA), was developed in the late 1990s and

Gentofte Hospital HR-Research Unit and Department of Gastroenterology F, Gentofte Hospital, University of Copenhagen, Copenhagen, Denmark

Corresponding author:

Astrid Collatz Schyum, Ved Mønten 10, 01, 110, DK-2300 Copenhagen S, Denmark.

Email: astridcs2@hotmail.com 
has shown sensitivities and specificities close to EMA. Besides, tTG immunoglobulin (Ig) G may be usable in diagnosing $\mathrm{CD}$ in individuals with $\operatorname{IgA}$ deficiency. ${ }^{3,13}$ The current literature on diagnosis of CD reflects controversies among clinicians with respect to preferred screening tests, screening algorithms and the role played by HLA typing in the diagnosis of CD. Are we approaching a scenario in which $\mathrm{CD}$ can be diagnosed or excluded in adults without performing a small intestinal biopsy? We will address these questions in the present systematic overview of recent papers on serological testing for $\mathrm{CD}$ in adults.

\section{Material and methods}

Search strategy in MeSH database (limits: 2006-2013)

Initial search: celiac disease $\rightarrow$ add to searchbuilder with $A N D$, serologic tests $\rightarrow$ add to searchbuilder and search PubMed: celiac disease AND serologic tests, which brought us 107 hits. First, the articles were chosen by their title alone. This was followed by a selection based on abstracts.

The same method was used in the following searches: celiac disease AND serologic tests AND gliadin (22 hits), celiac disease AND serologic tests AND transglutaminase (42 hits), celiac disease AND histocompatibilitytesting (33 hits). Searching the same way for EMA and deamidated gliadin brought no results in MeSH.

\section{Search strategy on PubMed (limits: 2006-2012)}

Initial search: celiac disease and serologic tests brought us 173 hits. First, the articles were selected based on their titles and subsequently based on their abstracts. Second search: celiac disease and deamidated gliadin (108 hits), celiac disease and serologic tests and deamidated gliadin (22 hits), celiac disease and serologic tests and transglutaminase (96 hits), celiac disease and EMA (192 hits), celiac disease and serologic tests and EMA (34 hits), celiac disease and HLA (530 hits). The selection of articles was conducted as explained above.

In selecting studies, cohort and case-control studies were included, and only those published between 2006 and 2013 were selected as to practically include all studies of the DGP assay. Studies including only children were excluded as we wanted to focus on serological testing in adults. Likewise studies with unclear study design or those for which the diagnosis of $\mathrm{CD}$ was not confirmed by biopsy were excluded. These criteria were met by the studies listed in Table $1 .{ }^{10,11,17-24}$ Sensitivities, specificities, study prevalences together with positive predictive values (PPV) and negative predictive values (NPV) are shown whenever these where reported (definition of the terms according to Jørgensen et al., ${ }^{25}$ Table 2). Because information of normal distribution of data was rarely given, it was decided to use nonparametric median and ranges. ${ }^{25}$

All studies used met the requirement that all diagnoses of CD were finally confirmed by a biopsy and not by serologic testing alone, as biopsy is considered necessary by many for diagnosing CD. ${ }^{2,8,15,16}$ Studies that, before testing, had taken IgA deficiency into account as being a possible condition among the participants (by measuring total $\operatorname{Ig} \mathrm{A}$ ) were preferred to avoid this being a source of error in the final results, as IgA deficiency is common in CD patients. ${ }^{24}$

\section{Results}

EMA

Five studies (three case-control and two cohort studies) studied EMA assays: Four studied EMA IgA tests $^{11,21-23}$ and one studied a EMA IgG test. ${ }^{24}$

EMA IgA. The median sensitivity was $0.835(0.61-0.937)$. The median specificity was high; $1(0.98-1) . P P V$ was $0.82(0.644-1)$. NPV was high; 0.969 (0.944 and 0.994).

EMA IgG. The study by Villalta et al. ${ }^{24}$ tested the performance of an EMA IgG assay. The only value reported was the sensitivity of the test: 0.758 .

(See Table 1 for further information about the studies.)

tTG. Nine different studies (four case-control and five cohort studies) studied tTG assays: Eight studied tTG $\operatorname{Ig} \mathrm{A}^{10,11,18-23}$ and two tTG $\operatorname{IgG} .^{10,24}$

tTG IgA. The median sensitivity was the highest of all the examined (single) assays; $0.93(0.76-0.968)$. The median specificity was $0.952(0.909-0.98)$. $P P V$ was $0.937(0.286-0.969)$. The median $N P V$ was 0.968 (0.963-0.996).

tTG IgG. Only two values of sensitivity were reported; 0.414 and 0.842 . There was only reported one specificity value that was relatively high (0.988). No $P P V \mathrm{~s}$ or $N P V \mathrm{~s}$ were reported.

(See Table 1 for further information about the studies.)

$D G P$

Eight different studies (five case-control and three cohort studies) studied DGP assays: Seven studied DGP IgA tests ${ }^{10,11,17-21}$ and seven studied DGP $\operatorname{IgG}$ tests. ${ }^{10,11,17-20,24}$ 
Table 1. Summary of the examined studies

\begin{tabular}{|c|c|c|c|c|}
\hline Author & Study design & No. of participants & Selection criteria & Biopsy \\
\hline Veermersch et al. ${ }^{10}$ & Case-control & $\begin{array}{l}\text { Cases: Total of } 86 \text { newly diagnosed CD patients. Blood } \\
\text { samples analyzed from the time of diagnosis. } \\
\text { Controls: Total of } 741 \text { consecutive non-CD patients. }\end{array}$ & $\begin{array}{l}\text { Newly diagnosed CD. No previ- } \\
\text { ously known diagnosis of CD or } \\
\text { treatment with GFD. }\end{array}$ & All participants. \\
\hline Sugai et al. ${ }^{18}$ & Case-control & $\begin{array}{l}\text { Cases: Total of } 92 \text { consecutive CD patients. Blood } \\
\text { samples analyzed from the time of diagnosis. } \\
\text { Controls: } 113 \text { non-CD patients undergoing gas- } \\
\text { troscopy because of GI problems. Hereunder } 29 \\
\text { patients with newly diagnosed Crohn's disease. }\end{array}$ & $\begin{array}{l}\text { Cases: suspected CD (symptoms of } \\
\text { CD), SBB taken. } \\
\text { Controls: Non-CD. GI disorder. }\end{array}$ & $\begin{array}{l}\text { All participants except for } \\
\text { the } 29 \text { patients with } \\
\text { Crohn's disease. }\end{array}$ \\
\hline Sugai et al. ${ }^{19}$ & Cohort & $\begin{array}{l}\text { Total of } 161 \text { in high-risk group suspected of GI dis- } \\
\text { order; } 518 \text { patients in low-risk group undergoing } \\
\text { routine endoscopy because of nonspecific } \\
\text { symptoms. }\end{array}$ & $\begin{array}{l}\text { Individuals referred to endoscopy } \\
\text { because of suspected small } \\
\text { bowel disorder. No previously } \\
\text { diagnosed GI disorder. }\end{array}$ & All participants. \\
\hline Niveloni et al. ${ }^{20}$ & Cohort & $\begin{array}{l}\text { Total of } 141 \text { consecutive patients with suspected GI } \\
\text { disorder. }\end{array}$ & $\begin{array}{l}\text { Symptoms of GI disorder. Referred } \\
\text { for endoscopy. No previously } \\
\text { diagnosed GI disorder or } \\
\text { treatment. }\end{array}$ & All participants. \\
\hline Volta et al. ${ }^{11}$ & Case-control & $\begin{array}{l}\text { Cases: Total of } 128 \text { patients with CD. Blood samples } \\
\text { analyzed from the time of diagnosis. } \\
\text { Controls: Total of } 134 \text { patients with differential } \\
\text { diagnosis to CD. }\end{array}$ & $\begin{array}{l}\text { Biopsy proven confirmation or } \\
\text { exclusion of CD. Controls have } \\
\text { well-known differential } \\
\text { diagnosis. }\end{array}$ & $\begin{array}{l}\text { All cases. } \\
\quad 43 / 134 \text { controls. }\end{array}$ \\
\hline Kaukinen et al. ${ }^{21}$ & Case-control & $\begin{array}{l}\text { Cases: } 53 \mathrm{CD} \text { patients ( } 44 \text { with untreated } C D \text { and nine } \\
\text { with nonresponsive } C D \text { despite long-term treat- } \\
\text { ment). } \\
\text { Controls: } 46 \text { non-CD patients with abdominal } \\
\text { symptoms. }\end{array}$ & Gl symptoms. SBB performed. & All participants. \\
\hline Hopper et al. ${ }^{22}$ & Case-control & $\begin{array}{l}\text { Cases: } 77 \text { CD patients. Blood samples analyzed from } \\
\text { time of diagnosis. } \\
\text { Controls: Total of } 1923 \text { individuals without CD. }\end{array}$ & $\begin{array}{l}\text { Consecutive patients referred for } \\
\text { gastroscopy. No previously } \\
\text { diagnosed CD, coagulopathy, } \\
\text { Gl bleeding or suspected } \\
\text { carcinoma. }\end{array}$ & All participants. \\
\hline Dahle et al. ${ }^{23}$ & Cohort & $\begin{array}{l}176 \text { patients referred for endoscopy and SBB because } \\
\text { of GI symptoms ( } 79 \text { of these known to have } C D \text { ). }\end{array}$ & $\begin{array}{l}\text { Endoscopy and SBB performed. } \\
\text { No previously diagnosed CD. } \\
\text { No GFD. }\end{array}$ & All participants. \\
\hline Tonutti et al. ${ }^{17}$ & Case-control & $\begin{array}{l}\text { Cases: Total of } 144 \text { patients with CD, nine on GFD. The } \\
\text { rest: blood samples analyzed from the time of } \\
\text { diagnosis. } \\
\text { Controls: Total of } 129 \text { non-CD individuals ( } 60 \\
\text { healthy blood donors and } 69 \text { patients with } \mathrm{Gl} \\
\text { disorders other than CD). }\end{array}$ & $\begin{array}{l}\text { Cases: biopsy proven CD. } \\
\text { Controls: No CD. }\end{array}$ & $\begin{array}{l}\text { All cases. Controls } \\
\text { unknown. }\end{array}$ \\
\hline Villalta et al. ${ }^{24}$ & Case-control & $\begin{array}{l}\text { Cases: } 34 \text { CD patients. Blood samples analyzed from } \\
\text { the time of diagnosis. } \\
\text { Controls: Six individuals without CD. }\end{array}$ & CD diagnosed by SBB. & All participants. \\
\hline
\end{tabular}

CD: celiac disease; GFD: gluten-free diet; Gl: gastrointestinal; SBB: small bowel biopsy.

DGP IgA. The median sensitivity was the second highest of all the assays studied: $0.91(0.69-0.984)$. The median specificity was high; $0.969(0.903-0.98)$ like the median PPV; $0.945(0.892-0.989)$ and $N P V ; 0.972$ (0.852-0.989).

DGP IgG. Here the median sensitivity was high as well: $0.882(0.754-0.967)$. The median specificity was $0.996(0.985-1)$. The median $P P V$ was high as well: $1(0.982-1)$ and $N P V$ was 0.959 (0.869-0.97).
(See Table 1 for further information about the studies.)

\section{Combination assays (DGP and tTG)}

Three of the cohort studies tested combination assays: ${ }^{19,20,23}$ DGP IgA and $\operatorname{IgG}$ combined with tTG $\operatorname{IgA}$ and $\operatorname{IgG}$, DGP IgA in combination with tTG IgA, DGP IgG combined with tTG IgA and finally the combination of DGP IgA and IgG (Table 3). All combination assays showed high sensitivity, specificity, PPVs 
Table 2. Median (range) for assays tested in different studies

\begin{tabular}{llllll}
\hline Assay & Sensitivity $^{\mathrm{a}}$ & Specificity $^{\mathrm{a}}$ & PPV $^{\mathrm{a}}$ & NPV $^{\mathrm{a}}$ & Reference no. $^{\mathrm{b}}$ \\
\hline EMA IgA & $0.835(0.61-0.937)$ & $1(0.98-1)$ & $0.82(0.644-1)$ & $0.969(0.944-0.994)$ & $11,21-23$ \\
EMA IgG & 0.758 & - & - & - & 24 \\
tTG IgA & $0.93(0.76-0.968)$ & $0.952(0.909-0.98)$ & $0.937(0.286-0.969)$ & $0.968(0.963-0.996)$ & $10,11,18-23$ \\
tTG IgG & $0.628(0.414-0.842)$ & 0.988 & - & - & 10,24 \\
DGP IgA & $0.91(0.69-0.984)$ & $0.969(0.903-0.98)$ & $0.945(0.892-0.989)$ & $0.972(0.852-0.989)$ & $10,11,17-21$ \\
DGP IgG & $0.882(0.754-0.967)$ & $0.996(0.985-1)$ & $1(0.982-1)$ & $0.959(0.869-0.97)$ & $10,11,17-20,24$ \\
\hline
\end{tabular}

The finding of medians is based on sensitivities, specificities, PPVs (positive predictive values) and NPVs (negative predictive values) specified in a $95 \%$ confidence interval (CI). The assays are: EMA (endomysial antibodies), tTG (tissue transglutaminase antibodies) and DGP (deamidated gliadin peptide

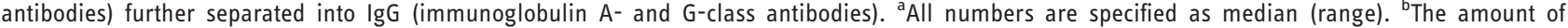
numbers is equal to the amount of references used in calculating sensitivity, specificity, PPV and NPV of the different assays.

Table 3. Median (range) for combination assays tested in different studies

\begin{tabular}{llllll}
\hline Assay & \multicolumn{1}{c}{ Sensitivity $^{\mathrm{a}}$} & \multicolumn{1}{c}{ Specificity $^{\mathrm{a}}$} & PPV $^{\mathrm{a}}$ & NPV $^{\mathrm{a}}$ & Reference no. $^{b^{2}}$ \\
\hline DGP (IgA and IgG)+ tTG (IgA and IgG) & $1(0.91-1)$ & $0.855(0.8-0.91)$ & $0.928(0.903-0.952)$ & $1(1)$ & $19,20,23$ \\
DGP IgA + tTG IgA & $0.968(0.936-1)$ & $0.958(0.926-0.99)$ & $0.947(0.909-0.984)$ & $0.98(0.959-1)$ & 19,20 \\
DGP IgG + tTG IgA & $0.953(0.905-1)$ & $0.988(0.975-1)$ & $0.984(0.967-1)$ & $0.97(0.94-1)$ & 19,20 \\
DGP IgA and IgG & $0.952(0.87-0.983)$ & $0.96(0.988-1)$ & $0.99(0.983-1)$ & $0.979(0.97-0.988)$ & $19,20,23$ \\
\hline
\end{tabular}

The finding of medians is based on sensitivities, specificities, PPVs (positive predictive values) and NPVs (negative predictive values) specified in a $95 \%$ confidence interval (CI). The assays are: tTG (tissue transglutaminase antibodies) and DGP (deamidated gliadin peptide antibodies) further separated into IgG (immunoglobulin A- and G-class antibodies). ${ }^{a}$ All numbers are specified as median (range). ${ }^{\text {b}}$ The amount of numbers is equal to the amount of references used in calculating sensitivity, specificity, PPV and NPV of the different assays.

and NPVs with medians above 0.9 except for DGP (IgA and $\operatorname{IgG})+$ tTG $(\operatorname{IgA}$ and $\operatorname{IgG})$, which had the lowest specificity: $0.855(0.8-0.91){ }^{19,20,23}$ On the other hand, this assay had the highest sensitivity and NPV (1 in both studies where the sensitivity was reported). DGP IgG + tTG IgA had the highest specificity: 0.988 $(0.975-1) \cdot{ }^{19,20}$ DGP $(\operatorname{IgA}+\operatorname{IgG})$ had the highest PPV: $0.99(0.983-1){ }^{19,20,23}$ DGP $(\operatorname{IgA})+\mathrm{tTG}(\operatorname{IgA})$ had the second highest sensitivity: $0.968(0.936-1)$ and NPV: $0.98(0.959-1){ }^{19,20}$

\section{Prevalences in cohort-studies}

In the three cohort studies by Sugai et al., ${ }^{19}$ Niveloni et al. $^{20}$ and Dahle et al. ${ }^{23}$ included in this work, all patients were referred for gastroscopy because of suspected gastrointestinal (GI)/malabsorption disorder. All patients underwent duodenal biopsy. ${ }^{19,20,23}$ The prevalence of CD in the three studies extends from $39.1 \%$ to $44.9 \%$, which is very high in consecutive patient materials, however. PPVs and NPVs are reported in two of the studies, ${ }^{19,20}$ and here all PPVs and NPVs are higher than those of the case-control studies, except for the NPVs of tTG IgA and the PPVs of DGP IgG, which are comparable with the case-control results. The same cut-off of $20 \mathrm{U} / \mathrm{ml}$ was used in testing all assays ${ }^{19,20,23}$ except for AGA, for which the cut-off was $25 \mathrm{U} / \mathrm{ml}$. Dahle et al. $^{23}$ used the cut-off of $5 \mathrm{U} / \mathrm{ml}$ for the tTG IgA assay.

\section{$H L A-D Q$}

HLA DQ2/DQ8 typing is typically used to exclude CD in patients when misdiagnosis is suspected or in patients on a gluten-free diet for whom the diagnosis is unclear and gluten-challenge is not possible. ${ }^{9,14-16,26,27}$ Some find HLA DQ2 or DQ8 negativity to be sufficient to rule out a diagnosis of $\mathrm{CD}^{6,13,13,26,27}$ as the tissue types are considered necessary for the development of CD. ${ }^{28-30}$

In a study by Karell et al., ${ }^{31} 61 / 1008 \mathrm{CD}$ patients were found to be missing HLA DQ2 and DQ8 hetero-dimers. Of these 61 patients, 57 patients expressed one of the alleles of the HLA DQ2 dimer. Kupfer et $a .^{32}$ found that individuals expressing only one allele of the DQ2 dimer are at low risk of developing CD. Hadithi et al. ${ }^{30}$ compared the efficiency of serologic measuring and HLA DQ testing in the diagnosis of $\mathrm{CD}$, respectively, and concluded that the expression of HLA DQ2 or DQ8 is necessary but not sufficient for the development of $\mathrm{CD}$. Likewise, the authors find that a biopsy is redundant in cases for which both serological tests and HLA DQ2 or DQ8 are positive. 
Others find that a biopsy is still an absolute necessity in diagnosing $\mathrm{CD}$ regardless of other test results. ${ }^{26,33-35}$

Torres et al. ${ }^{28}$ investigated whether HLA G (coding for major histocompatibility complex (MHC) class I molecules) is associated with CD. All 24 CD patients studied expressed HLA G while none of the nine non$\mathrm{CD}$ individuals did the same. In addition, raised levels of serum HLA G in patients with other diagnosis, e.g. Down syndrome or autoimmune thyroid disease, were observed while there was found low or negative values in $\mathrm{CD}$ patients on a gluten-free diet for more than five years. Fabris et al. ${ }^{29}$ confirmed the results of Torres et al. Trynka et al. recently found 13 new CD risk loci, bringing the number of known loci (including HLA) to $40 .^{36}$

\section{Discussion}

IgA tests. EMA has a low sensitivity: $(0.835$, (0.610.937)), compared to earlier studies that rate EMA as the best test (comparable with tTG). ${ }^{6,26,33}$ Dahle et al. $^{23}$ explain the low sensitivity of EMA $\operatorname{IgA}$ in their study by the relatively old age of all participants included (half of them are 50 years or above) as old age has been connected to less true-positive EMA results compared to studies that include younger adults. Despite the generally low sensitivity, EMA IgA has the highest specificity of all assays together with a high NPV, and therefore it is usable to identify CD, e.g. in persons in a low-risk group. tTG IgA has the highest sensitivity of all (single) assays and a high specificity overruled only by DGP IgA, which also has the second highest sensitivity. This indicates that the assay is suitable for identifying persons with $\mathrm{CD}$ and can be used for screening purposes. The high values match the results from other studies that also find tTG IgA to be the most successful test together with EMA. 3,6,8,26,34,37

$\lg G$ tests. There is insufficient data about EMA IgG and tTG IgG in the selected studies to make firm conclusions. Earlier studies have shown both low sensitivities and low specificities of the two assays. ${ }^{8,33}$ Kaukinen et al. ${ }^{21}$ show an impaired performance of AGA assays when non-CD patients have symptoms compatible with CD. In their study, 54\% of the non-CD control group was tested positive for AGA.

DGP IgG shows superior performance compared to DGP IgA and other IgG assays. It has a relatively high sensitivity, a high specificity and the highest PPV of all (single) assays. DGP IgG could be especially effective in persons with $\operatorname{Ig}$ A deficiency (concluded by others as well ${ }^{8,10,20,24}$ ) and it could therefore be used in routine testing as a supplement to a tTG IgA test.

All combination assays have higher sensitivities than each single assay alone (Table 3). Almost all combinations have higher specificities exceeded only by DGP IgG (0.996 (0.985-1)) and EMA IgA (1 (0.98-1)). All the PPVs of the combinations are higher than those of AGA and EMA IgA (single assays) while all the NPVs are higher than those of the single assays alone.

When comparing the different study designs, cohort studies are preferable over case-control studies if the prevalence rates of $\mathrm{CD}$ in the cohort are realistic (Table 2). Cohort studies use consecutively chosen patients with symptoms compatible with $\mathrm{CD}$ and provide reliable predictive values if prevalence rates are relevant. None of the patients involved in the included cohort studies were previously diagnosed with or excluded from having CD (except for Dahle et al. ${ }^{23}$ (see Table 1)) and thereby expectation bias is avoided, which can be an issue in case-control studies if they are not properly blinded. Cases are specifically chosen and have all been diagnosed with $\mathrm{CD}$, while the control group often consists of healthy individuals without symptoms or other patient groups. Biopsy is omitted (or not reported) in some control groups ${ }^{11,17}$ and therefore the presence of $\mathrm{CD}$ cannot be excluded with certainty.

Comparison of the three cohort studies included in this work by Sugai et al., ${ }^{19}$ Niveloni et al. ${ }^{20}$ and Dahle et al. ${ }^{23}$ show prevalences of CD extending from $39.1 \%$ to $44.9 \%$. This is a very high prevalence of $\mathrm{CD}$ in consecutive materials even in highly specialized practice, although reservations due to overlapping patient material must be taken into account for Sugai et al. ${ }^{19}$ and Niveloni et al. ${ }^{20}$ The PPVs and NPVs reported in these two studies ${ }^{19,20}$ show that all PPVs and NPVs are higher in these study populations than those of the case-control studies, except for the NPVs of tTG IgA and the PPVs of DGP IgG, which are comparable with the case-control results. The cut-off values of the assays are the same in the three cohort studies, which justifies comparison of the study results.

For many years it has been known that there is a strong association between the tissue types HLA DQ8 and DQ2 and the risk of developing CD. ${ }^{5,6}$ Karell et al. ${ }^{31}$ confirm this, as 57 patients lacking the HLA DQ2 dimer express one of the alleles. It is therefore important that HLA typing be performed on each allele separately to avoid individuals being incorrectly placed into nonrisk groups. In many cases a HLA test alone is considered sufficient for excluding persons from the at-risk group. ${ }^{6,13,26,27}$ However, the Karell et al. study ${ }^{31}$ also exemplifies the opposite as CD in rare cases has been seen without the occurrence of HLA DQ2 or DQ8. However, the possibility that 
these rare cases represent misdiagnosis must be considered. The connection between CD and HLA $\mathrm{G}^{28,29}$ and its role in tissue typing in the clinic must be further investigated. It seems fair to conclude that if CD-like disease develops in HLA DQ2/DQ8-negative individuals, then it is very rare.

In conclusion, tTG IgA and DGP IgG show the best performance from a clinical diagnostic standpoint. It seems as preferable as combining two or more assays (especially DGP (IgA and IgG) + tTG (IgA and IgG) or DGP (IgA and $\operatorname{IgG})$ ) as this shows both very high sensitivities and specificities (Table 3) compared to the single assays as concluded by others. ${ }^{7,9,14,16}$ However, data derive from three studies only, and additional prospective studies as well as cost-benefit analyses are necessary before final recommendations can be made. In addition, more cohort studies should be conducted using DGP assays (both alone and combined with tTG) to confirm or refute the usefulness of these tests compared to other assays (tTG or EMA alone). Based on the above studies in adults, it is still premature to recommend a diagnostic strategy for CD in adults that omits duodenal biopsy. Such a strategy seems feasible in subgroups of children with CD. Here intestinal biopsies can be avoided in one-third or more of seropositive individuals in the presence of high CD-specific antibody titers, gluten-dependent symptoms and the presence of HLA DQ2 and/or HLA DQ8 ${ }^{13}$ Further cohort studies in adults, also taking predictive values of antibody titers into consideration, are anticipated, preferably with designs allowing evaluation of diagnostic strategies that aim to omit intestinal biopsies.

\section{Funding}

This research received no specific grant from any funding agency in the public, commercial, or not-for-profit sectors.

\section{Conflict of interest}

The authors declare that there is no conflict of interest.

\section{References}

1. Tjon JM, van Bergen J and Koning F. Celiac disease: How complicated can it get? Immunogenetics 2010; 62: 641-651.

2. Craig D, Robins G and Howdle PD. Advances in celiac disease. Curr Opin Gastroenterol 2007; 23: 142-148.

3. Fasano A and Catassi C. Clinical practice. Celiac disease. $N$ Engl J Med 2012; 367: 2419-2426.

4. Chang $\mathrm{M}$ and Green PH. Genetic testing before serologic screening in relatives of patients with celiac disease as a cost containment method. J Clin Gastroenterol 2009; 43: 43-50.

5. Piccini B, Vascotto M, Serracca L, et al. HLA-DQ typing in the diagnostic algorithm of celiac disease. Rev Esp Enferm Dig 2012; 104: 248-254.
6. Kaukinen K, Lindfors $\mathrm{K}$ and Collin P. Coeliac disease - a diagnostic and therapeutic challenge. Clin Chem Lab Med 2010; 48: 1205-1216.

7. van der Windt DA, Jellema P, Mulder CJ, et al. Diagnostic testing for celiac disease among patients with abdominal symptoms: A systematic review. JAMA 2010; 303: 1738-1746.

8. Leffler DA and Schuppan D. Update on serologic testing in celiac disease. Am $J$ Gastroenterol 2010; 105: 2520-2524.

9. Lindfors K, Koskinen O and Kaukinen K. An update on the diagnostics of celiac disease. Int Rev Immunol 2011; 30: 185-196.

10. Vermeersch P, Geboes K, Marien G, et al. Diagnostic performance of IgG anti-deamidated gliadin peptide antibody assays is comparable to IgA anti-tTG in celiac disease. Clin Chim Acta 2010; 411: 931-935.

11. Volta U, Granito A, Fiorini E, et al. Usefulness of antibodies to deamidated gliadin peptides in celiac disease diagnosis and follow-up. Dig Dis Sci 2008; 53: 1582-1588.

12. Reeves GE, Squance ML, Duggan AE, et al. Diagnostic accuracy of coeliac serological tests: A prospective study. Eur J Gastroenterol Hepatol 2006; 18: 493-501.

13. Husby S, Koletzko S, Korponay-Szabo IR, et al. European Society for Pediatric Gastroenterology, Hepatology, and Nutrition guidelines for the diagnosis of coeliac disease. J Pediatr Gastroenterol Nutr 2012; 54: $136-160$.

14. Volta U and Villanacci V. Celiac disease: Diagnostic criteria in progress. Cell Mol Immunol 2011; 8: 96-102.

15. Evans KE and Sanders DS. What is the use of biopsy and antibodies in coeliac disease diagnosis? J Intern Med 2011; 269: 572-581.

16. Ludvigsson JF and Green PH. Clinical management of coeliac disease. J Intern Med 2011; 269: 560-571.

17. Tonutti E, Visentini D, Picierno A, et al. Diagnostic efficacy of the ELISA test for the detection of deamidated anti-gliadin peptide antibodies in the diagnosis and monitoring of celiac disease. J Clin Lab Anal 2009; 23: 165-171.

18. Sugai E, Vázquez H, Nachman F, et al. Accuracy of testing for antibodies to synthetic gliadin-related peptides in celiac disease. Clin Gastroenterol Hepatol 2006; 4: 1112-1117.

19. Sugai E, Moreno ML, Hwang HJ, et al. Celiac disease serology in patients with different pretest probabilities: Is biopsy avoidable? World J Gastroenterol 2010; 16: 3144-3152.

20. Niveloni S, Sugai E, Cabanne A, et al. Antibodies against synthetic deamidated gliadin peptides as predictors of celiac disease: Prospective assessment in an adult population with a high pretest probability of disease. Clin Chem 2007; 53: 2186-2192.

21. Kaukinen K, Collin P, Laurila K, et al. Resurrection of gliadin antibodies in coeliac disease. Deamidated gliadin peptide antibody test provides additional diagnostic benefit. Scand J Gastroenterol 2007; 42: 1428-1433.

22. Hopper AD, Hadjivassiliou M, Hurlstone DP, et al. What is the role of serologic testing in celiac disease? A 
prospective, biopsy-confirmed study with economic analysis. Clin Gastroenterol Hepatol 2008; 6: 314-320.

23. Dahle C, Hagman A, Ignatova S, et al. Antibodies against deamidated gliadin peptides identify adult coeliac disease patients negative for antibodies against endomysium and tissue transglutaminase. Aliment Pharmacol Ther 2010; 32: 254-260.

24. Villalta D, Tonutti E, Prause C, et al. IgG antibodies against deamidated gliadin peptides for diagnosis of celiac disease in patients with IgA deficiency. Clin Chem 2010; 56: 464-468.

25. Jørgensen T, Christensen E and Kampmann JP. Klinisk forskningsmetode. 2.edit. ed. Danmark: Munksgaard, 2005, pp.46-49.

26. Green PH and Cellier C. Celiac disease. N Engl J Med 2007; 357: 1731-1743.

27. Dubois PC and van Heel DA. Translational mini-review series on the immunogenetics of gut disease: Immunogenetics of coeliac disease. Clin Exp Immunol 2008; 153: 162-173.

28. Torres MI, López-Casado MA, Luque J, et al. New advances in coeliac disease: Serum and intestinal expression of HLA-G. Int Immunol 2006; 18: 713-718.

29. Fabris A, Segat L, Catamo E, et al. HLA-G 14 bp deletion/insertion polymorphism in celiac disease. Am J Gastroenterol 2011; 106: 139-144.

30. Hadithi $M$, von Blomberg BM, Crusius JB, et al. Accuracy of serologic tests and HLA-DQ typing for diagnosing celiac disease. Ann Intern Med 2007; 147: 294-302.
31. Karell K, Louka AS, Moodie SJ, et al. HLA types in celiac disease patients not carrying the DQA1*05DQB1*02 (DQ2) heterodimer: Results from the European Genetics Cluster on Celiac Disease. Hum Immunol 2003; 64: 469-477.

32. Kupfer SS and Jabri B. Pathophysiology of celiac disease. Gastrointest Endosc Clin N Am 2012; 22: 639-660.

33. Rostom A, Dube C, Cranney A, et al. The diagnostic accuracy of serologic tests for celiac disease: A systematic review. Gastroenterology 2005; $128(4$ Suppl 1): S38-S46.

34. Lewis NR and Scott BB. Meta-analysis: Deamidated gliadin peptide antibody and tissue transglutaminase antibody compared as screening tests for coeliac disease. Aliment Pharmacol Ther 2010; 31: 73-81.

35. Evans KE, Hadjivassiliou M and Sanders DS. Is it time to screen for adult coeliac disease? Eur J Gastroenterol Hepatol 2011; 23: 833-838.

36. Trynka G, Hunt KA, Bockett NA, et al. Dense genotyping identifies and localizes multiple common and rare variant association signals in celiac disease. Nat Genet 2011; 43: 1193-1201.

37. Alessio MG, Tonutti E, Brusca I, et al. Correlation between $\operatorname{IgA}$ tissue transglutaminase antibody ratio and histological finding in celiac disease. $J$ Pediatr Gastroenterol Nutr 2012; 55: 44-49. 